\title{
Lennard-Jones 团簇最低能量构型的预测
}

\author{
赖向京*，许如初，黄文奇 \\ 华中科技大学计算机科学与技术学院, 武汉 430074 \\ *通讯作者, Email: laixiangjing@yahoo.com.cn \\ 收稿日期: 2010-05-18; 接受日期: 2010-09-09 \\ doi: $10.1007 / \mathrm{s} 11426-011-4280-4$
}

\begin{abstract}
摘要 针对 Lennard-Jones(LJ)团簇的结构优化问题, 在前人工作的基础上, 提出了一个新 的无偏优化算法, 即 DLS-TPIO (dynamic lattice searching method with two-phase local search and interior operation)算法. 对 $\mathrm{LJ}_{2-650}, \mathrm{LJ}_{660}, \mathrm{LJ}_{665-680}$ 这 666 个实例进行了优化计算. 为其中每个 实例所找到的构型其势能均达到了剑桥团簇数据库中公布的最好记录. 对 $\mathrm{LJ}_{533}$ 与 $\mathrm{LJ}_{536}$ 这两 个算例,所达到的势能则优于先前的最好记录. 在 DLS-TPIO 算法中,采用了内部操作,两阶段 局部搜索方法以及动态格点搜索方法. 在优化的前一阶段,内部操作将若干能量较高的表面 原子移入团簇的内部, 从而降低团簇的能量, 并使其构型逐渐地变为有序. 与此同时, 两阶段 局部搜索方法指导搜索进入更有希望的构型区域。这种做法显著地提高了算法的成功率. 在 优化的后一阶段, 借用动态格点搜索方法对团簇表面原子的位置作进一步优化, 以再一次降 低团簇的能量. 另外, 为识别二十面体构型的中心原子, 本文给出了一个简单的新方法. 相 比于文献中一些著名的无偏优化算法, DLS-TPIO 算法具有较高的计算速度与成功率.
\end{abstract}

关键词

全局优化

Lennard-Jones 团簇 内部操作 两阶段局部搜索 动态格点搜索

\section{1 引言}

团簇的结构优化问题也即团簇最低能量结构的 预测问题. 它是计算化学领域的重要研究课题之一. 确定团簇的最低能量结构有利于我们了解真实团簇 的许多物理与化学性质 ${ }^{[1 ~ 3]}$. 然而, 由于其构型空间 随团簇尺度的增加而呈指数型增长, 这类问题的求 解通常是 NP (nondeterministic polynomial-time) 难度 的. 因此, 对于这类问题, 发展高效的全局优化算法 具有重要的理论和现实意义.

在过去的二十年中, LJ 团簇受到广泛而深入的 研究, 事实上, 它已经成为全局优化算法的试金石. 其势能函数可表示如下:

$$
E_{\mathrm{LJ}}=4 \varepsilon \sum_{i=1}^{N-1} \sum_{j=i+1}^{N}\left[\left(\frac{\sigma}{r_{i j}}\right)^{12}-\left(\frac{\sigma}{r_{i j}}\right)^{6}\right]
$$

其中 $N$ 表示原子个数, $r_{i j}$ 表示原子 $i$ 与原子 $j$ 之间的 距离, $\varepsilon=1.0, \sigma=1.0$. 由式(1)可知, LJ 团簇的结构 优化实质上是一个无约束的连续优化问题.

在目前的文献中,已有大量的算法用于求解这一 优化问题. 具有代表性的包括: basin-hopping 及其变 形 ${ }^{[4 \sim 7]}$, simulated annealing $(\mathrm{SA})^{[8,9]}$, hierarchical greedy algorithm(HGA) $)^{[10]}$, genetic algorithms $(\mathrm{GAs})^{[11 \sim 15]}$, conformation space annealing algorithm(CSA $)^{[16]}$, adaptive immune optimization algorithm(AIOA) ${ }^{[17]}$, random tunnel algorithm(RTA $)^{[18]}$, heuristic algorithm with surface and interior operator(HA-SIO $)^{[19]}$, dynamic lattice searching algorithm(DLS)及其变形 ${ }^{[20 ~ 23]}$, simple greedy method with energy-based perturbation and a small population (EP-SGM) ${ }^{[24]}$, 及其他一些算法 ${ }^{[25,26]}$.

求解该问题的算法可分为两类, 一类是有偏算 法, 另一类是无偏算法 ${ }^{[22]}$. 有偏算法是指预先构建与 
具体图形相关的格点, 例如, 二十面体格点, 然后基 于所建的格点进行优化操作. 这类算法具有较快的 计算速度, 但由于构型空间受到限制, 很难保证所得 解的最优性. 使用这类算法, 能为 $N=2 \sim 1610$ 范围内 的大部分实例找到已知的最低能量构型 ${ }^{[15,27 \sim 29]}$. 无 偏算法是指从随机生成的构型开始, 基于随机扰动 的方式进行优化操作 ${ }^{[22]}$. 使用这类算法, 许多实例的 最低能量构型第一次被找到. 例如, 1997 年, Wales 和 Doye ${ }^{[4]}$ 用 basin-hopping 算法找到了 $\mathrm{LJ}_{69}, \mathrm{LJ}_{78}, \mathrm{LJ}_{107}$ 的 最低能量构型; 1999 年, Leary ${ }^{[7]}$ 用 monotonic sequence basin-hopping (MSBH)算法找到了 $\mathrm{LJ}_{98}$ 的最低能量构 型; 2006 年, Takeuchi ${ }^{[19]}$ 用 HA-SIO 算法找到了 $\mathrm{LJ}_{506}$, $\mathrm{LJ}_{521}, \mathrm{LJ}_{537}, \mathrm{LJ}_{538}, \mathrm{LJ}_{541}$ 的最低能量构型. 相比于有偏 算法, 无偏算法具有更大的搜索空间, 因此, 也具有 更高的可靠性.

当前, 对于团簇的结构优化问题, DLS 方法 ${ }^{[20]}$ 与 两阶段局部搜索方法 ${ }^{[30]}$ 是两种特别有效的优化技术. DLS 方法合适地利用了团簇的结构信息, 从而具有 很高的计算速度, 是当前最有效的优化方法之一. 另 一方面, 研究表明, 两阶段局部搜索通过利用一个变 换的势能函数, 能有效地提高算法的效率 ${ }^{[31]}$. 事实上, 它已成功地应用于许多优化问题 ${ }^{[30 \sim 32]}$.

为进一步发展团簇的结构优化算法, 本文基于 DLS 算法, 两阶段局部搜索方法及一个简单的内部 操作(将在 2.1 节中介绍), 提出了一种新的无偏优化 算法, 即 DLS-TPIO 算法. 为了检测该算法的性能, 它被用于 $\mathrm{LJ}_{2-650}, \mathrm{LJ}_{660}, \mathrm{LJ}_{665-680}$ 的优化. 实验结果表 明, DLS-TPIO 算法具有较高的计算速度和成功率.

\section{2 计算方法}

DLS-TPIO 算法基本思想是充分利用内部操作, 两阶段局部搜索方法, 动态格点搜索方法的优点, 以 提高算法的计算效率. 这些方法在 DLS-TPIO 算法中 的作用将于第 3 节中讨论.

DLS-TPIO算法起始于一个随机生成的局部最小 值构型, 然后循环地执行内部操作与两阶段局部搜 索, 直到团簇的能量连续 10 次未得到改进. 最后, 对所 得构型执行动态格点搜索. 此外, 对于大尺度团簇 (尺度大于等于 500), 创建空心操作以及第二次动态 格点搜索也将执行. 下面给出了 DLS-TPIO 算法的详 细描述.

\section{1 内部操作}

一般情况下, 对处于平衡状态的 LJ 团簇, 其内 部的原子比表面的原子具有更小的能量. 出于这种 考虑, Takeuchi ${ }^{[19]}$ 提出了一个内部操作, 即将若干个 能量较高的表面原子移入团簇的内部, 以降低这些 原子的能量. 2008 年, 邵学广等人 ${ }^{[22]}$ 提出了该内部操 作的一个变形.

本文给出的内部操作与 Takeuchi 和邵学广等人 提出的类似, 即将能量最大的前 $m$ 个原子随机地放 在以团簇几何中心为球心, 0.5 为半径的球内, 其中 $1 \leqslant m \leqslant 5$, 第 $i$ 个原子的能量 $E(i)$ 可用式(2)计算. 与 前面两种内部操作相比, 该内部操作更易于实现.

$$
E(i)=4 \cdot \sum_{j \neq i}^{N}\left(\frac{1}{r_{i j}^{12}}-\frac{1}{r_{i j}^{6}}\right)
$$

\section{2 两阶段局部搜索方法}

两阶段局部搜索方法 ${ }^{[30]}$ 由两个阶段组成，第一 阶段使用一个修改的势能函数 $E_{\mathrm{LJ}}^{\mathrm{mod}}$ 执行局部搜索, 以指导搜索进入更有希望的构型区域；第二阶段以 第一阶段的结果作为输入, 用原始的势能函数 $E_{\mathrm{LJ}}$ 执 行局部搜索，以得到其局部最小值.

在本文算法中, 修改的势能函数可表示如下:

$$
\begin{aligned}
E_{\mathrm{LJ}}^{\mathrm{mod}}= & \sum_{i=1}^{N-1} \sum_{j=i+1}^{N}\left[\frac{1}{r_{i j}^{10}}-\frac{1}{r_{i j}^{5}}+\mu \cdot r_{i j}\right. \\
& \left.+\beta \cdot\left(\max \left\{0, r_{i j}^{2}-(\eta \cdot R)^{2}\right\}\right)^{2}\right]
\end{aligned}
$$

其 中 $\mu=0.1, \beta=2.0, \quad \eta \in\{1.0,1.35\}, R=(N /$ $17.77)^{1 / 3}+1.0^{[22]}, r_{i j}$ 为原子 $i$ 与原子 $j$ 之间的距离, $N$ 为团簇尺度.

对于 LJ 团簇的结构优化, Locatelli 与 Schoen 在 文献[30]中也给出了一个修改的势能函数. 在当前的 研究中, 我们对其进行了修改, 从而得到了式(3). 这 两个势能函数的不同之处在于团簇直径的估计值具 有不同的表达形式. 在式(3)中, 团簇直径的估计值 用 $\eta \cdot R$ 表示, 因 $R$ 只取决于团簇尺度 $N$, 故当 $\eta$ 被固 定时, 团簇直径的估计值将随 $N$ 自适应地取值; 而在 Locatelli 与 Schoen 给出的势能函数中, 团簇直径的 估计值直接用 $D$ 表示, 且对于不同的 $N$, 需根据经验 分别设置它的值. 相比之下, 使用式(3)是更方便的.

在上述势能函数(3)中, $\beta \cdot\left(\max \left\{0, r_{i j}{ }^{2}-(\eta \cdot R)^{2}\right\}\right)^{2}$ 表示团簇直径的惩罚项, $\mu \cdot r_{i j}$ 表示两原子间距离的 
惩罚项. 由于势能函数(3)对两个相距较远的两原子 进行惩罚, 故它支持球状的构型. 而这种构型和大部 分 LJ 团簇的最低能量构型是一致的. 因此, 势能函 数(3)的使用能有效地指导搜索计算, 从而提高算法 的成功率.

\section{3 动态格点搜索算法}

DLS 算法由邵学广与程龙㺵等人 ${ }^{[20]}$ 提出. 该算 法起始于一个随机生成的局部最小值构型, 然后循 环地执行动态格点构建, 动态格点搜索, 局部搜索, 直到无法找出更低能量的构型. 动态格点构建是指 为一个添加的原子找出团簇表面所有可能的平衡位 置, 这些平衡位置和团簇中 $N_{\mathrm{mov}}\left(N_{\mathrm{mov}}\right.$ 为预先设定的 正整数) 个能量最高的原子所在的位置一起被称为动 态格点, 这些动态格点组成了一个动态格子. 动态格 点搜索是指用一个简单的贪婪算法搜索动态格子以 得到若干个能量较低的候选解. 有关动态格点搜索 算法的详细描述可在文献[20]中找到.

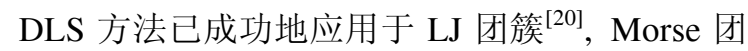
簇 ${ }^{[33]}, \mathrm{C}_{60}$ 分子团簇 ${ }^{[34]}, \mathrm{Ag}$ 原子团簇 ${ }^{[35]}$. 实验结果表 明, DLS 方法是高效、无偏的.

\section{4 创建空心操作}

对于许多大尺度 $\mathrm{LJ}$ 实例, 其最低能量构型是无 中心原子的二十面体 ${ }^{[36]}$. 因此, 本文算法包含创建空 心的操作, 即将团簇的中心原子随机地移到团簇的 表面. 为了识别二十面体构型的中心原子, 本文引入 原子密度的概念.

原子 $i$ 的原子密度定义为:

$$
D(i)=\sum_{\substack{j=1(\neq i) \\ d_{i j} \leqslant \sqrt[6]{2}}}^{N} \frac{1}{d_{i j}^{3}}
$$

其中 $d_{i j}$ 表示原子 $i$ 与原子 $j$ 之间的距离, $N$ 为团簇尺 度.

对于二十面体构型的团簇, 因为处于结构内层 的原子受到外层原子的挤压, 内层原子间的距离比 外层原子间的距离更小 ${ }^{[37]}$, 故根据式(4)可知, 越内 层的原子, 原子密度越大. 因此, 原子密度最大的原 子通常是中心原子.

\section{5 局部搜索方法}

本文使用有限内存的拟牛顿法 (L-BFGS) ${ }^{[38]}$ 作为
局部搜索方法. 研究表明, 对于团簇的结构优化问 题, L-BFGS 方法具有很强的局部搜索能力, 而且通 常优于共轭梯度法 ${ }^{[39]}$. 事实上, 它已广泛地应用于团 簇的结构优化问题.

\subsection{DLS-TPIO 算法的描述}

(1)在以坐标原点为球心, $R$ 为半径的球内随机地 生成初始构型, 然后用 L-BFGS 方法执行局部搜索, 并将更新失败次数 NoImprove 赋值为 0 . 其中 $R=(N / 17.77)^{1 / 3}+1.0, N$ 为团簇尺度.

(2)对当前团簇执行内部操作, 随后执行两阶段 局部搜索.

(3)若团簇的能量有所降低, 则更新构型(即将所 得构型替换原来的构型), 并将 NoImprove 赋值为 0 , 然后回到(2); 否则保留原来的构型, 并将 NoImprove 增加 1 .

(4) 若更新失败次数 NoImprove $<10$, 则回到(2), 否则转到(5).

(5) 以当前构型作为初始构型, 执行动态格点搜 索程序.

(6)若所得团簇的能量小于等于已知的最低能量 或者团簇的尺度小于 500 , 则算法终止, 否则执行 (7).

(7)对当前团簇执行创建空心操作, 即将其中原 子密度最大的原子随机地放在团簇的表面.

(8)以所得构型作为初始构型, 执行第二次动态 格点搜索程序.

(9)算法终止.

在上述算法中, 若团簇的尺度小于 309 , 则内部 操作移动的原子数 $m$ 取值为 1 , 否则 $m$ 的取值为 1 5 之间的一个随机数. 此外, 对通常被认为是难例的 $\mathrm{LJ}_{38}, \mathrm{LJ}_{75-77}, \mathrm{LJ}_{102-104}, \mathrm{LJ}_{188-192}, \mathrm{LJ}_{236-238}$, 式(3)中的参 数 $\eta$ 取值为 1.0 ; 对其他实例, $\eta$ 取值为 1.35 . 图 1 则 是上述算法的流程图.

\section{3 结果与讨论}

DLS-TPIO 算法用 C 语言实现, 其所有计算在 $2.4 \mathrm{GHz}$ CPU, $1 \mathrm{G}$ 内存的 PC 机上完成. DLS-TPIO 算 法中所用的参数值列在表 1 中, 其中各参数的含义与 文献[20]中的相同. 本研究中, 考虑到计算成本, 我 们只优化了 $N=2 \sim 650,660,665 \sim 680$ 范围内的团簇. 对于表 2 4 中的每个算例, DLS-TPIO 算法独立地执 


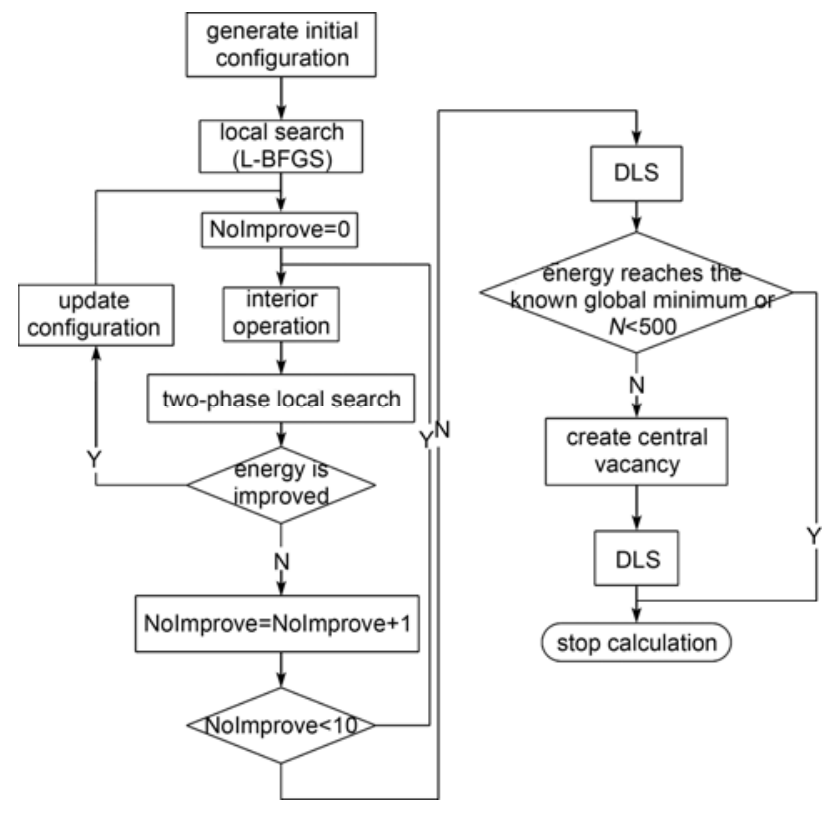

图 1 DLS-TPIO 算法的流程图

\section{行了 250 次.}

通过对上述范围内的团簇进行优化, DLS-TPIO 算法找到了所有的已知最低能量构型(最新的能量值 列表可在剑桥团簇数据库中找到: http: //www-wales. ch.cam.ac.uk/CCD.html). 对于 $\mathrm{LJ}_{533}$ 与 $\mathrm{LJ}_{536}$ 这两个算 例, 所达到的势能优于先前的最好记录. 表 5 中, 我 们将这两个新的最低能量与其先前的最低能量进行 了比较. 另外, 本文所得新的最低能量构型已存放于 剑桥团簇数据库中.

找出最低能量构型平均所需的局部搜索次数通 常被用于评价算法的计算速度 ${ }^{[6,10,17,19 \sim 24]}$. 局部搜索 次数越少, 则计算速度越快. 对一些有代表性的算 例, 表 2 列出了 DLS-TPIO 算法的局部搜索次数, 并 将其与其他几种算法的结果进行了比较. 从表 2 可 知, 除 $\mathrm{LJ}_{50}, \mathrm{LJ}_{98}, \mathrm{LJ}_{100}$ 外, 对其中所有算例, DLS-

表 1 DLS-TPIO 算法中所用的参数值

\begin{tabular}{ccccc}
\hline$N$ & $N_{\text {mov }}$ & $N_{\mathrm{p}}$ & $N_{\text {best }}$ & $N_{\text {try }}$ \\
\hline $13 \sim 49$ & 10 & 92 & 4 & 100 \\
$50 \sim 150$ & 15 & 252 & 5 & 400 \\
$151 \sim 200$ & 15 & 252 & 10 & 500 \\
$201 \sim 309$ & 20 & 492 & 10 & 700 \\
$310 \sim 450$ & 30 & 642 & 15 & 1000 \\
$451 \sim 561$ & 40 & 812 & 15 & 1000 \\
$562 \sim 665$ & 60 & 812 & 15 & 1500 \\
$666 \sim 680$ & 80 & 812 & 20 & 1500 \\
\hline
\end{tabular}

TPIO 算法的局部搜索次数比其他几种算法的均更少. 例如，对于 $\mathrm{LJ}_{75}$, HA-SIO 算法 ${ }^{[19]}$ 与 DLS 算法 ${ }^{[20]}$ 的局 部搜索次数分别是 DLS-TPIO 算法的约 12 倍, 7 倍. 对于 $\mathrm{LJ}_{500}, \mathrm{HA}-\mathrm{SIO}$ 算法 ${ }^{[19]}$ 与 $\mathrm{HGA}$ 算法 ${ }^{[10]}$ 的局部搜 索次数分别是 DLS-TPIO 算法的约 75 倍, 89 倍.

对于 $N=100 \sim 580$ 范围内的实例, 图 2 给出了 DLS-TPIO 算法找出其最低能量构型所用的局部搜索 次数. 从图像可以看出, 对其中的所有实例, 局部搜 索次数均不超过 $7 \times 10^{3}$, 而且, 对其中大部分实例, 局部搜索次数不超过 $10^{3}$. 此外, 在 $N=188,236,350$, 484,578 附近, 分别出现了 1 个波峰. 这表明 $\mathrm{LJ}_{188}$, $\mathrm{LJ}_{236}, \mathrm{LJ}_{350}, \mathrm{LJ}_{484}, \mathrm{LJ}_{578}$ 是 DLS-TPIO 算法的难例. 另 一方面, 通过将图 2 与文献[19]中的图 2 进行比较, 不难发现, 对于 $N=100 \sim 561$ 范围内的大部分实例, HA-SIO 算法 ${ }^{[19]}$ 所需的局部搜索次数是 DLS-TPIO 算 法的几倍至几百倍. 这些结果表明 DLS-TPIO 算法的 计算速度是相当快的.

CPU 时间通常也是评价算法性能的一个标 准 ${ }^{[19 \sim 23]}$. 对于 $N=100 \sim 580$ 范围内的实例, 图 3 给出 了 DLS-TPIO 算法找出其最低能量构型所消耗的 $\mathrm{CPU}$ 时间. 从图 3 可以看出, 对其中所有实例, CPU 时间均少于 $10^{4} \mathrm{~s}$, 且对其中大部分实例, CPU 时间少 于 $10^{3}$ s. 此外, 对于 $\mathrm{LJ}_{N}(N=100,200,300,400,500$, $561)$, DLS-TPIO 算法的平均 CPU 时间分别是 0.53 , $0.467,1.08,3.95,20.07,8.78 \mathrm{~min}$. 然而, 根据 HA-SIO 算法 ${ }^{[19]}$ 的结果，相应的 CPU 时间分别是 $1.3 ， 16,29$, $1059,2016,470 \mathrm{~min}$ (计算在 $3 \mathrm{GHz}$ CPU, $1 \mathrm{G}$ 内存的 计算机上执行).

成功率是评价算法性能的另一个标准 ${ }^{[6,17,20 \sim 24]}$. 对于一些有代表性的算例, 表 3 中给出了 DLS-TPIO

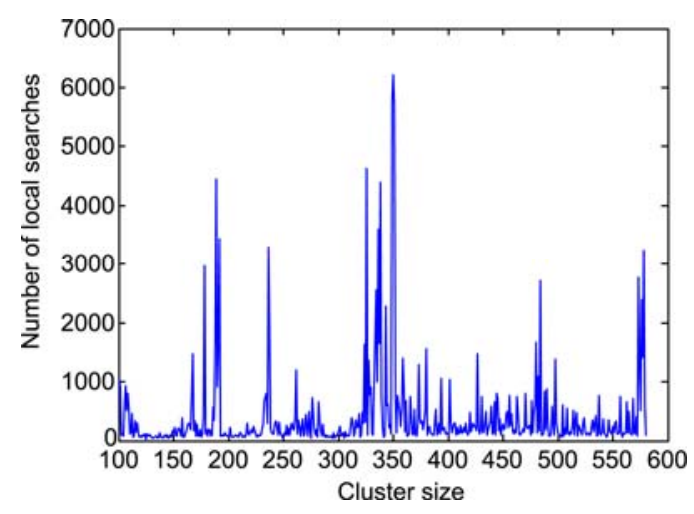

图 2 搜索最优构型所需的局部搜索次数 
表 2 搜索最低能量构型所需的局部搜索次数

\begin{tabular}{|c|c|c|c|c|c|c|c|}
\hline$N$ & DLS-TPIO & $\mathrm{HA}-S I O^{[19]}$ & $\operatorname{DLS}^{[20]}$ & $\mathrm{AIOA}^{[17]}$ & EP-SGM $^{[24]}$ & $\mathrm{MSBH}^{[6]}$ & $\mathrm{HGA}^{[10]}$ \\
\hline 38 & 56 & 2352 & 3240 & 3046 & 1739 & 2875 & \\
\hline 50 & 96 & 51 & 270 & 537 & 264 & 460 & \\
\hline 75 & 357 & 4374 & 2586 & 43000 & 86000 & 152000 & 9259 \\
\hline 98 & 1138 & 3312 & 597 & 32000 & 27200 & 180000 & 5660 \\
\hline 100 & 659 & 844 & 610 & 9304 & 2525 & 9128 & \\
\hline 102 & 95 & 3881 & 7733 & 31000 & 13880 & 36028 & \\
\hline 150 & 115 & 267 & 1398 & & 3230 & & \\
\hline 188 & 1614 & 7320 & 12234 & & & & \\
\hline 200 & 124 & 2371 & 4494 & 140000 & 56550 & & \\
\hline 236 & 3299 & 16216 & 30875 & & & & \\
\hline 250 & 185 & 4611 & 11347 & & & & \\
\hline 300 & 112 & 2017 & 14875 & & & & \\
\hline 400 & 208 & 42600 & & & & & \\
\hline 450 & 336 & & & & & & \\
\hline 500 & 695 & 52462 & 70000 & & & & 62000 \\
\hline 561 & 213 & 9419 & & & & & \\
\hline
\end{tabular}

表 3 DLS-TPIO 算法的成功率

\begin{tabular}{cc||cc}
\hline$N$ & 成功率 & $N$ & 成功率 \\
\hline $38^{\mathrm{b})}$ & $232 / 250$ & $200^{\mathrm{a})}$ & $176 / 250$ \\
$50^{\mathrm{a})}$ & $154 / 250$ & $236^{\mathrm{b}}$ & $6 / 250$ \\
$75^{\text {b) }}$ & $41 / 250$ & $250^{\mathrm{a})}$ & $114 / 250$ \\
$98^{\mathrm{b})}$ & $16 / 250$ & $300^{\mathrm{a})}$ & $186 / 250$ \\
$100^{\mathrm{a})}$ & $26 / 250$ & $400^{\mathrm{a}}$ & $134 / 250$ \\
$102^{\mathrm{b})}$ & $167 / 250$ & $450^{\mathrm{a}}$ & $85 / 250$ \\
$150^{\mathrm{a})}$ & $165 / 250$ & $500^{\mathrm{a})}$ & $51 / 250$ \\
$188^{\mathrm{b})}$ & $12 / 250$ & $561^{\mathrm{a}}$ & $154 / 250$ \\
\hline
\end{tabular}

a) 最优构型为二十面体; b)最优构型为非二十面体

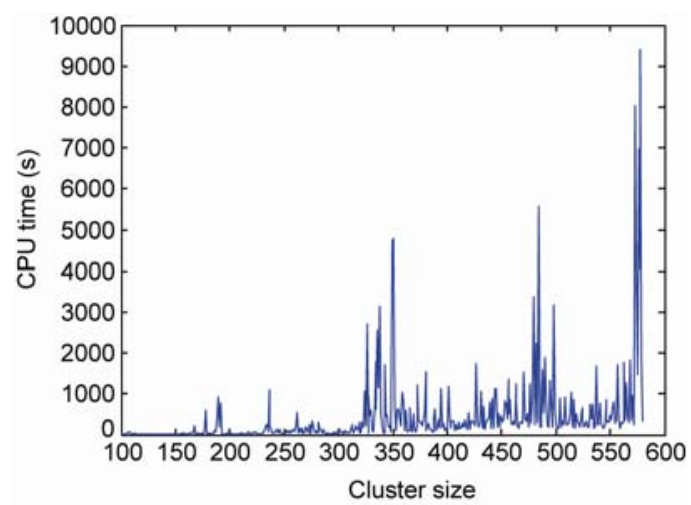

图 3 搜索最优构型所消耗的计算时间

算法的成功率. 从表 3 可以看出, 对最优构型为二十 面体的算例, DLS-TPIO 算法能获得很高的成功率. 例如, 对 $\mathrm{LJ}_{300}$, 成功率是 $186 / 250$. 而且, 对最优构型 为非二十面体的算例, DLS-TPIO 算法的成功率也并
不低. 例如, 对 $\mathrm{LJ}_{98}$ 与 $\mathrm{LJ}_{188}$, 成功率分别是 $16 / 250$, $12 / 250$. 值得注意的是, 对于 $\mathrm{LJ}_{38}$ 与 $\mathrm{LJ}_{102}$, DLS-TPIO 算法具有很高的成功率; 然而, 对大部分随机优化算 法而言, 它们则是难例.

对于大尺度 LJ 团簇的优化, DLS-IO (dynamic lattice searching method with interior operation)算法 ${ }^{[22]}$ 是目前最有效的无偏优化算法之一; 为了说明 DLSTPIO 算法的效率, 表 4 中, 我们将 DLS-TPIO 算法与 DLS-IO 算法 ${ }^{[22]}$ 的结果进行了比较. 从表 4 可以看出, 在平均局部搜索次数, 平均 $\mathrm{CPU}$ 时间以及成功率方 面, DLS-TPIO 算法均优于 DLS-IO 算法 ${ }^{[22]}$. 例如, 对 于 $\mathrm{LJ}_{665}, \mathrm{DLS}-\mathrm{IO}$ 算法 ${ }^{[22]}$ 的平均局部搜索次数, 平均 CPU 时间, 成功率分别是 $152205,209.17 \mathrm{~h}, 0.1 \%$; 而 DLS-TPIO 算法的结果分别是 $2649,2.893 \mathrm{~h}, 6 \%$.

在 DLS-TPIO 算法中, 内部操作, 两阶段局部搜 索, 动态格点搜索方法分别起了重要的作用. 在优化 的前一阶段, 内部操作将若干能量较高的表面原子 移入团簇的内部, 从而降低团簇的能量, 并使其构型 逐渐变为有序 ${ }^{[22]}$. 与此同时, 两阶段局部搜索方法引 导搜索进入更有希望的构型区域. 这种方式极大地 提高了算法的成功率. 在优化的后一阶段, 使用动态 格点搜索方法对表面原子的位置作进一步优化, 再 一次降低了团簇的能量.

需要指出的是, DLS-TPIO 算法仍然是无偏的. 尽管修改的势能函数(3)支持球状的构型, 但由于团簇 的初始构型是随机生成的, 内部操作也具有随机性, 
表 4 DLS-TPIO 算法与 DLS-IO 算法的比较

\begin{tabular}{|c|c|c|c|c|c|c|}
\hline \multirow{2}{*}{$N$} & \multicolumn{3}{|c|}{ DLS-TPIO } & \multicolumn{3}{|c|}{ DLS-IO ${ }^{[22]}$} \\
\hline & 平均局部搜索次数 & 平均 CPU 时间(h) & 成功率 $(\%)$ & 平均局部搜索次数 & 平均 CPU 时间(h) & 成功率 $(\%)$ \\
\hline 500 & 695 & 0.284 & 20.4 & 9943 & 10.76 & 0.6 \\
\hline 561 & 213 & 0.146 & 60.2 & 9302 & 15.09 & 0.8 \\
\hline 660 & 39745 & 43.3 & 0.4 & 124028 & 181.40 & 0.1 \\
\hline 665 & 2649 & 2.893 & 6 & 152205 & 209.17 & 0.1 \\
\hline 670 & 375 & 0.405 & 43.2 & 21208 & 43.02 & 0.6 \\
\hline
\end{tabular}

DLS-TPIO 与 DLS-IO 算法分别在 $2.4 \mathrm{GHz}$ CPU, $1 \mathrm{G}$ 内存与 $2.8 \mathrm{GHz}$ CPU, $1 \mathrm{G}$ 内存的计算机上执行

表 5 新的与先前的最低能量

\begin{tabular}{ccc}
\hline$N$ & 新的最低能量 & 先前的最低能量 \\
\hline 533 & -3629.299922 & -3628.252883 \\
536 & -3651.941851 & -3651.779047 \\
\hline
\end{tabular}

$\mathrm{LJ}_{533}$ 与 $\mathrm{LJ}_{536}$ 先前的最低能量值分别来自文献[15], [19]

因此其他形状的构型也有机会得到. 以 $\mathrm{LJ}_{100}$ 为例, 在 我们实验中, 二十面体构型, 十面体构型, 面心体构 型均能得到. 另一方面, 非二十面体最优构型的获得 同时也表明了 DLS-TPIO 算法的无偏性.

此外, 实验结果表明, 式(3)中 $\eta$ 的取值对团簇构 型的形成具有较大影响. 以 $\mathrm{LJ}_{75}$ 为例, 当 $\eta=1.0$ 时, 算法的成功率是 $41 / 250$, 而当 $\eta=1.35$ 时, 成功率只 有 $4 / 250$. 这一结果的主要原因也许是, 当 $\eta$ 取不同 的值时, 团簇内层原子受到外层原子的挤压力大小 也不同. 随着 $\eta$ 减小，内层原子受到的挤压力则增大.

最后，我们指出，尽管 DLS-TPIO 算法对于 $N \leqslant 680$ 范围内的大部分算例均具有较高的计算速度, 但对以十面体为最低能量构型的大尺度团簇 $\left(\mathrm{LJ}_{650-664}\right)$, 计算也是非常耗时的. 例如, 对于 $\mathrm{LJ}_{660}$,
找出最低能量构型所需的 CPU 时间达到 $43.3 \mathrm{~h}$. 因此, 对该算法做进一步的改进依然是需要的.

\section{4 结论与展望}

基于内部操作,两阶段局部搜索方法以及动态格 点搜索方法, 本文提出了一种新的无偏优化算法, 即 DLS-TPIO 算法. 使用该算法, 我们对 $N=2 \sim 650,660$, 665 680 范围内的 LJ 团簇进行了优化. DLS-TPIO 算 法为其中每个实例所找到的构型其势能均达到了剑 桥团簇数据库中公布的最好记录. 而且, 对 $\mathrm{LJ}_{533}$ 与 $\mathrm{LJ}_{536}$ 这两个算例, 所达到的势能则优于先前的最好 记录. 与文献中一些著名的无偏优化算法相比, DLSTPIO 算法具有较高的计算速度与成功率. 这些结果 表明，对于 LJ 团簇的结构优化问题, DLS-TPIO 算法 是相当有效的优化工具. 而且, 对于其他一些原子团 簇的结构优化, 如 Morse 团簇, 该算法也是适用的.

在将来的研究中, 我们希望将 DLS-TPIO 算法应 用于其他原子团簇的结构优化; 其中, 在两阶段局部 搜索期间，我们打算使用不同的团簇直径惩罚函数. 结果，倪海文及叶涛与作者进行了有益的讨论. 本工作得到国家自然科学基金(60773194,61070235)资助. 特 此一并致谢.

\section{参考文献}

1 Doye JPK. Physical perspectives on the global optimization of atomic clusters. In: Pinter JD, eds. Global Optimization: Scientific and Engineering Case Studies. Berlin: Springer-Verlag, 2006, 103-139

2 Cheng LJ, Feng Y, Yang J, Yang JL. Funnel hopping: Searching the cluster potential energy surface over the funnels. J Chem Phys, 2009, 130: 214112

3 Fa W, Luo CF, Dong JM. Bulk fragment and tubelike structures of $\mathrm{Au}_{N}(N=2-26)$. Phys Rev B, 2005, 72: 205428

4 Wales DJ, Doye JPK. Global optimization by basin-hopping and the lowest energy structures of Lennard-Jones clusters containing up to 110 
atoms. J Phys Chem A, 1997, 101: 5111-5116

5 Wales DJ, Scheraga HA. Global optimization of clusters, crystals and biomolecules. Science, 1999, 285: 1368-1372

6 Leary RH. Global optimization on funneling landscapes. J Global Optim, 2000, 18: 367-383

7 Leary RH. Tetrahedral global minimum for the 98-atom Lennard- Jones cluster. Phys Rev E, 1999, 60: R6320-R6322

8 Xue GL. Molecular conformation on the CM-5 by parallel two-level simulated annealing. J Global Optim, 1994, 4: 187-208

9 Wille LT. Simulated annealing and the topology of the potential energy surface of Lennard-Jones clusters. Comput Mater Sci, 2000, 17: $551-554$

10 Krivov SV. Hierarchical global optimization of quasiseparable systems: Application to Lennard-Jones clusters. Phys Rev E, 2002, 66: 025701

11 Deaven DM, Tit N, Morris JR. Structure optimization of Lennard-Jones clusters by a genetic algorithm. Chem Phys Lett, 1996, 256: 195-200

12 Wolf MD, Landman U. Genetic algorithms for structural cluster optimization. J Phys Chem A, 1998, 102: 6129-6137

13 Hartke B. Global cluster geometry optimization by a phenotype algorithm with niches: Location of elusive minima, and lower-order scaling with cluster size. J Comput Chem, 1999, 20: 1752-1759

14 Romero D, Barron C, Gomez S. The optimal geometry of Lennard- Jones clusters: 148-309. Comput Phys Commun, 1999, 123: 87-96

15 Xiang YH, Jiang HY, Cai WS, Shao XG. An efficient method based on lattice construction and the genetic algorithm for optimization of large Lennard-Jones clusters. J Phys Chem A, 2004, 108: 3586-3592

16 Lee J, Lee I, Lee J. Unbiased global optimization of Lennard-Jones clusters for $N \leqslant 201$ using the conformational space annealing method. Phys Rev Lett, 2003, 91: 080201

17 Cheng LJ, Cai WS, Shao XG. A connectivity table for cluster similarity checking in the evolutionary optimization method. Chem Phys Lett, 2004, 389: 309-314

18 Jiang HY, Cai WS, Shao XG. A random tunneling algorithm for the structural optimization problem. Phys Chem Chem Phys, 2002, 4: $4782-4788$

19 Takeuchi H. Clever and efficient method for searching optimal geometries of Lennard-Jones clusters. J Chem Inf Model, 2006, 46: 2066-2070

20 Shao XG, Cheng LJ, Cai WS. A dynamic lattice searching method for fast optimization of Lennard-Jones clusters. J Comput Chem, 2004, 25: $1693-1698$

21 Yang XL, Cai WS, Shao XG. A dynamic lattice searching method with constructed core for optimization of Lennard-Jones clusters. J Comput Chem, 2007, 28: 1427-2433

22 Shao XG, Yang XL, Cai WS. A dynamic lattice searching method with interior operation for unbiased optimization of large Lennard-Jones clusters. J Comput Chem, 2008, 29: 1772-1779

23 Wu X, Cai WS, Shao XG. A dynamic lattice searching method with rotation operation for optimization of large clusters. Chem Phys, 2009, 363: $72-77$

24 Cheng LJ, Cai WS, Shao XG. An energy-based perturbation and a taboo strategy for improving the searching ability of stochastic structural optimization methods. Chem Phys Lett, 2005, 404: 182-186

25 Pullan W. An unbiased population-based search for the geometry optimization of Lennard-Jones Clusters: $2 \leqslant N \leqslant 372$. $J$ Comput Chem, 2005, 26: 899-906

26 Cai WS, Shao XG. A fast annealing evolutionary algorithm for global optimization. J Comput Chem, 2002, 23: 427-435

27 Northby JA. Structure and binding of Lennard-Jones clusters: $13 \leqslant N \leqslant 147$. J Chem Phys, 1987, 87: 6166-6177

28 Xiang YH, Cheng LJ, Cai WS, Shao XG. Structural distribution of Lennard-Jones clusters containing 562 to 1000 atoms. J Phys Chem A, 2004 , 108: 9516-9520

29 Shao XG, Xiang YH, Cai WS. Structural transition from icosahedra to decahedra of Lennard-Jones clusters. J Phys Chem A, 2005, 109: 5193-5197

30 Locatelli M, Schoen F. Efficient algorithms for large scale global optimization: Lennard-Jones clusters. Comput Optim Appl, 2003, 26: $173-190$

31 Doye JPK, Leary RH, Locatelli M, Schoen F. The global optimization of Morse clusters by potential energy transformations. INFORMS J Comput, 2004, 16: 371-379

32 Cassioli A, Locatelli M, Schoen F. Global optimization of binary Lennard-Jones clusters. Optim Method Softw, 2009, 24: 819-835

33 Cheng LJ, Yang JL. Global minimum structures of Morse clusters as a function of range of the potential: $81 \leqslant N \leqslant 160 . J P h y s C h e m$ A, 2007, 111: 5287-5293 
34 Cheng LJ, Cai WS, Shao XG. Geometry optimization and conformational analysis of $\left(\mathrm{C}_{60}\right)_{N}$ clusters by using a dynamic lattice searching method. ChemPhysChem, 2005, 6: 261-266

35 Zhan H, Cheng LJ, Cai WS, Shao XG. Structural optimization of silver clusters from $\mathrm{Ag}_{61}$ to $\mathrm{Ag}_{120}$ by dynamic lattice searching method. Chem Phys Lett, 2006, 422: 358-362

36 Shao XG, Xiang YH, Cai WS. Formation of central vacancy in icosahedral Lennard-Jones clusters. Chem Phys, 2004, 305: 69-75

37 Liu HH, Jiang EY, Bai HL, Wu P, Li ZQ. Impact of atomic shells on the structure of clusters. Chem Phys Lett, 2005, 412: 195-199

38 Liu DC, Nocedal J. On the limited memory BFGS method for large scale optimization. Math Program, 1989, 45: 503-528

39 Doye JPK. The effect of compression on the global optimization of atomic clusters. Phys Rev E, 2000, 62: 8753-8761

\title{
Prediction of the lowest energy configuration for Lennard-Jones clusters
}

\section{LAI XiangJing, XU RuChu \& HUANG WenQi}

School of Computer Science and Technology, Huazhong University of Science and Technology, Wuhan 430074, China

\begin{abstract}
Based on the work of previous researchers, a new unbiased optimization algorithm, called dynamic lattice searching method with two-phase local search and interior operation (DLS-TPIO), is proposed in this paper. This algorithm is applied to the optimization of Lennard-Jones (LJ) clusters with $N=2-650,660,665-680$. For each case, the putative global minimum reported in the Cambridge Cluster Database (CCD) is successfully found. Furthermore, for $\mathrm{LJ}_{533}$ and $\mathrm{LJ}_{536}$, the potential energies obtained in this study are superior to the previous best results. In DLS-TPIO, the interior operation, two-phase local search method and dynamic lattice searching method are adopted. At the initial stage of the optimization, the interior operation reduces the energy of the cluster, and makes the configuration ordered gradually, by moving some surface atoms with high potential energy to the interior of the cluster. Meanwhile, two-phase local search method guides the search move to the more promising region of the configuration space. In this way the success rate of the algorithm is increased significantly. At the final stage of the optimization, in order to decrease energy of the cluster again, the positions of surface atoms are further optimized by using dynamic lattice searching method. In addition, a simple new method to identify the center atom of icosahedral configurations is also presented. Compared with some famous unbiased optimization methods in the literature, DLS-TPIO has high computing speed and success rate.
\end{abstract}

Keywords: global optimization, Lennard-Jones clusters, interior operation, two-phase local search, dynamic lattice searching 